\title{
Efeito do Espaçamento, Idade e Irrigação no Volume e Densidade Básica do Eucalipto
}

\author{
Jordão Cabral Moulin ${ }^{1}$, Marina Donária Chaves Arantes ${ }^{1}$, \\ José Geraldo Lima de Oliveira ${ }^{1}$, Eduardo Campinhos ${ }^{2}$, Fabrício Gomes ${ }^{1}$, \\ Graziela Baptista Vidaurre ${ }^{1}$
}

${ }^{1}$ Departamento de Ciências Florestais e da Madeira - DCFM, Universidade Federal do Espírito Santo - UFES, Jerônimo Monteiro/ES, Brasil

${ }^{2}$ Empresa Fibria, Aracruz/ES, Brasil

\begin{abstract}
RESUMO
O objetivo do trabalho foi avaliar a influência da idade, irrigação e espaçamento no volume e densidade básica da madeira de eucalipto. Os materiais utilizados foram dois clones Eucalyptus grandis $\times$ Eucalyptus urophylla com idades de 6 e 12 meses provenientes de plantios em duas regiões, uma irrigada e outra região não irrigada, com espaçamentos de $3 \times 0,5 ; 1,5 \times 2$, $3 \times 1 ; 3 \times 2$ e $3 \times 3 \mathrm{~m}$. O volume foi determinado conforme o método de Smalian, sendo determinada também a densidade básica ponderada. Os maiores valores de volume de madeira por hectare foram obtidos nos espaçamentos mais densos e na região irrigada. Nos espaçamentos mais amplos, houve maiores valores de densidade básica da madeira para o clone $\mathrm{A}$, no entanto, no clone $\mathrm{B}$ não houve variação em função do espaçamento. Ao comparar os valores médios de densidade para os clones entre região irrigada e não irrigada, os resultados foram iguais na grande maioria dos espaçamentos estudados.
\end{abstract}

Palavras-chave: biomassa florestal, tratamento silvicultural, qualidade da madeira.

\section{Effect of Spacing, Age and Irrigation on the Volume and Basic Density in Eucalyptus}

\begin{abstract}
This study aimed to evaluate the influence of age, irrigation and spacing, on the volume and basic density of Eucalyptus wood. We used two clones of Eucalyptus grandis $\times$ Eucalyptus urophylla with age of six and twelve months, sowed in two regions, one irrigated and other unirrigated, with spacing of $3 \times 0.5,1.5 \times 2,3 \times 1,3 \times 2$ and $3 \times 3 \mathrm{~m}$. The volume was estimated according to the method of Smalian as well as the weighted density. The larger volume of wood per hectare values were obtained in the close spacing and irrigated region. Wider spacing showed higher specific gravity values timber for clone A; however, clone B didn't change with spacing. The comparison of average density values for the clones in irrigated and non-irrigated areas, showed similar results in most studied spacings.
\end{abstract}

Keywords: forest biomass, silvicultural treatment, quality of wood. 


\section{INTRODUÇÃO}

Entre os diversos fatores condicionantes da produção florestal, o espaçamento de plantio, a irrigação e a idade de corte exercem papéis fundamentais no estabelecimento, condução da floresta e custos de produção, uma vez que influenciam a taxa de crescimento das árvores e as práticas de implantação, manejo e colheita. Estes fatores ainda podem influenciar na qualidade da madeira.

Para implantação de uma floresta com características necessárias para atender a um determinado mercado de base madeireira, faz-se necessário a realização de um planejamento para verificar e analisar qual espaçamento deve ser empregado, para que a madeira formada neste plantio possa atender às exigências do mercado.

O espaçamento possui forte relação com as características de crescimento do eucalipto, como na área basal da árvore, o que ocasiona o aumento volumétrico desta, além de possuir influência na qualidade da madeira, idade de corte e, consequentemente, nos custos de produção (Brasil \& Ferreira, 1971; Schonau \& Coetzee, 1989).

De acordo com Wimmer et al. (2002), a irrigação altera a velocidade de crescimento da planta e algumas propriedades da madeira, como a densidade básica. A produtividade e a qualidade da madeira também estão relacionadas à idade de corte das árvores, principalmente quando se trata de espécies de rápido crescimento e de ciclos curtos de rotação (Mello et al., 1976).

Verificando a importância do espaçamento de plantio, irrigação e idade no crescimento da árvore e na qualidade da madeira, o presente trabalho possui objetivo de avaliar o volume e a densidade básica da madeira de dois clones de eucalipto em diferentes espaçamentos e idades, ambos em região irrigada e não irrigada.

\section{MATERIAL E MÉTODOS}

\subsection{Procedência do material, caracterização da região e amostragem}

Foram utilizados dois clones do híbrido de Eucalyptus grandis $\times$ Eucalyptus urophylla provenientes da Empresa Fibria Celulose S.A., unidade de Aracruz, Estado do Espírito Santo. A região do experimento em Aracruz localiza-se nas coordenadas geográficas a $19^{\circ} 49^{\prime} 15^{\prime \prime} \mathrm{S}$ de latitude e $40^{\circ} 05^{\prime} 20^{\prime \prime} \mathrm{W}$ de longitude. O solo da região é classificado como argissolo amarelo típico textura arenosa/ média fase relevo plano. A temperatura mínima e máxima do ar foi 21,11 e $24,89^{\circ} \mathrm{C}$, respectivamente, com média de $23^{\circ} \mathrm{C}$. A precipitação variou de 0,01 a $0,22 \mathrm{~mm}$, com média de 0,12 mm (FIBRIA, 2013).

O material em estudo, com idade de seis e doze meses, foi proveniente de diferentes espaçamentos e em duas áreas, uma irrigada e outra não irrigada. Para que o presente estudo pudesse traçar um perfil consistente da caracterização da madeira de eucalipto, foram amostradas três árvores por espaçamento de cada clone, idade e região, ou seja, 3 árvores $\times$ 5 espaçamentos $\times 2$ clones $\times 2$ idades $\times 2$ regiões, obtendo 120 árvores.

Para caracterização da madeira foram retirados seis discos com $2,5 \mathrm{~cm}$ de espessura, nas posições de $1,30 \mathrm{~m}$ do solo, $0 \%, 25 \%, 50 \%, 75 \%$ e $100 \%$ da altura comercial da árvore até atingir $5 \mathrm{~cm}$ de diâmetro, os quais foram utilizados para realização da análise química da madeira com uma amostra composta de cada árvore. No Quadro 1 está o esquema de implantação dos híbridos nos diversos espaçamentos e diferentes áreas.

Quadro 1. Esquema de implantação do povoamento para os tratamentos, clones, espaçamentos e irrigação.

Frame 1. Deployment scheme of the stand for treatments, clones, spacing and irrigation.

\begin{tabular}{|c|c|c|}
\hline $\begin{array}{l}\text { Material } \\
\text { Genético }\end{array}$ & $\begin{array}{c}\text { Espaçamento } \\
\text { (m) }\end{array}$ & $\begin{array}{c}\text { Irrigação } \\
(\mathrm{S} / \mathrm{I})\end{array}$ \\
\hline A & \multirow{2}{*}{$3 \times 3$} & \multirow{10}{*}{ I } \\
\hline B & & \\
\hline A & \multirow{2}{*}{$3 \times 2$} & \\
\hline B & & \\
\hline A & \multirow{2}{*}{$3 \times 1$} & \\
\hline B & & \\
\hline A & \multirow{2}{*}{$3 \times 0,5$} & \\
\hline B & & \\
\hline A & \multirow{2}{*}{$1,5 \times 2$} & \\
\hline B & & \\
\hline A & \multirow{2}{*}{$3 \times 3$} & \multirow{10}{*}{ S } \\
\hline B & & \\
\hline A & \multirow{2}{*}{$3 \times 2$} & \\
\hline B & & \\
\hline B & \multirow{2}{*}{$3 \times 1$} & \\
\hline A & & \\
\hline A & \multirow{2}{*}{$3 \times 0,5$} & \\
\hline B & & \\
\hline A & \multirow{2}{*}{$1,5 \times 2$} & \\
\hline B & & \\
\hline
\end{tabular}

A: Clone A; B: Clone B; I: Região irrigada; S: Região não irrigada. 


\subsection{Volume do fuste da madeira}

As árvores foram submetidas à cubagem rigorosa, para o cálculo do volume individual, por meio do método de Smalian. A altura comercial $(\mathrm{Hc})$ foi considerada até o diâmetro mínimo de $5 \mathrm{~cm}$, com casca. Adicionalmente, foram medidas as espessuras da casca para a determinação dos volumes com e sem casca, além das estimativas de incremento volumétrico das árvores.

Para caracterização da madeira foram retirados seis discos com $2,5 \mathrm{~cm}$ de espessura, nas posições de $1,30 \mathrm{~m}$ do solo, e a $0,25,50,75$ e $100 \%$ da altura comercial da árvore, os quais foram utilizados para determinação da densidade básica. Para realização da análise anatômica da madeira, utilizou-se amostra obtida na base da árvore, e para análise química da madeira foi utilizada uma amostra composta dos discos obtidos ao longo do fuste da árvore.

\subsection{Densidade básica da madeira}

Para a determinação da densidade básica, nas árvores de 6 e 12 meses de idade foi utilizado o disco inteiro em função das pequenas dimensões do tronco das árvores. A densidade básica da madeira foi determinada conforme a NBR 11941 (ABNT, 2003). Após a obtenção da densidade básica em cada posição, foi determinada a densidade básica pela média ponderada, pelo emprego do volume dos toretes das secções obtidas, Equação 1.

$\operatorname{DbmP}=\frac{\left(\operatorname{DBm}_{(0-25 \%)} \times \mathrm{V}_{(0-25 \%)}\right)+\ldots+\left(\operatorname{DBm}_{(75-100 \%)} \times \mathrm{V}_{(75-100 \%)}\right)}{\mathrm{V}_{(0-25 \%)}+\mathrm{V}_{(25-50 \%)}+\mathrm{V}_{(50-75 \%)}+\mathrm{V}_{(75-100 \%)}}$

em que:

DbmP: Densidade básica média ponderada $\left(\mathrm{g} . \mathrm{cm}^{-3}\right)$;

DBm: Densidade básica média entre as posições 0 e 25; 25 e 50; 50 e 75; 75 e 100(g.cm ${ }^{-3}$ );

V: Volume das seções entre as posições 0 e 25; 25 e 50; 50 e $75 ; 75$ e $100 \%\left(\mathrm{~m}^{3}\right)$.

\subsection{Análise estatística dos dados}

Para a região irrigada e não irrigada, foi realizada uma análise inicial de 20 tratamentos e 3 repetições no delineamento inteiramente casualizado, considerando os 20 tratamentos como um fatorial de 2 clones $\times 2$ idades $\times 5$ espaçamentos. Posteriormente foi realizada uma análise conjunta considerando as duas regiões, uma vez que a interação entre os quatro fatores foi significativa $(\mathrm{P}<0,05)$, optou-se em ignorar a estrutura fatorial; assim, as médias dos 40 tratamentos foram comparadas pelo teste de Skott-knott em nível de $5 \%$ de probabilidade.

\section{RESULTADOS E DISCUSSÃO}

\subsection{Volume do fuste de eucalipto}

Os valores médios de diâmetro da árvore a 1,30 m do solo (DAP), volumes de madeira e massa seca dos dois clones nas diferentes idades, espaçamentos e região irrigada e não irrigada podem ser visualizados na Tabela 1. Os valores de diâmetro a 1,30 m do solo, volume e massa seca por hectare diferiram estatisticamente $(\mathrm{P}<0,05)$. O DAP diminuiu com o adensamento do espaçamento, conforme Cardoso (1989) as árvores plantadas em espaçamentos densos possuem tendência de apresentar menor diâmetro do fuste, o que ocorre em função do pouco espaço para seu crescimento.

O maior volume foi do clone B com 12 meses no espaçamento de plantio $1,5 \times 2 \mathrm{~m}$ na região irrigada, sendo $55,02 \mathrm{~m}^{3}$ de madeira por hectare. Comparando os dois clones com a mesma idade, espaçamento e região, observou-se que os valores de volume do clone $\mathrm{B}$ foram superiores aos do clone A.

$\mathrm{O}$ volume de madeira por área aumentou com o adensamento do espaçamento para todas as condições de crescimento (Figura 1). Isto ocorreu por causa do maior número de árvores por área em menores espaçamentos, sendo o mesmo comportamento observado por Coelho et al. (1970), Balloni \& Simões (1980), Haygreen \& Bowyer (1989), Berger et al. (2002), Müller \& Couto (2006) e Morais (2006), que estudaram diferentes espécies e idades de Eucalyptus.

$\mathrm{O}$ volume de madeira por área foi maior na região irrigada nos dois clones e duas idades. $\mathrm{O}$ aumento de volume ocasionado pela irrigação é explicado por Haygreen \& Bowyer (1989), ao afirmarem que a irrigação possui papel de potencializar a taxa de crescimento das plantas. Tomazello (2006) obteve resultado semelhante, com maior produção em volume na área irrigada que na área não irrigada para a madeira do híbrido E. grandis $\mathrm{x}$ E. urophylla com idade de 7 anos e 1 mês.

A maior diferença de volume entre a região irrigada e não irrigada ocorreu no espaçamento mais amplo 
Tabela 1. Valores médios de diâmetro a $1,30 \mathrm{~m}$ do solo, volume e massa seca de madeira por hectare dos clones do híbrido E. grandis $\times$ E. urophylla nas diferentes idades, espaçamentos e regiões.

Table 1. Average values of diameter at $1.30 \mathrm{~m}$, volume and dry mass per hectare of hybrid clones E. grandis $\times$ E. urophylla in different ages, spacing and regions.

\begin{tabular}{|c|c|c|c|c|c|c|}
\hline Clone & Idade (meses) & $\begin{array}{c}\text { Espaçamento } \\
(\mathrm{m})\end{array}$ & $\operatorname{DAP}(\mathrm{cm})$ & $\begin{array}{l}\text { Volume }\left(\mathrm{m}^{3} /\right. \\
\text { ha com casca) }\end{array}$ & $\begin{array}{c}\text { Massa seca } \\
\text { (kg/ha) }\end{array}$ & Região \\
\hline \multirow{20}{*}{ A } & \multirow{10}{*}{6} & \multirow{2}{*}{$3 \times 3$} & $3,40 \mathbf{d}$ & $2,84 \mathbf{j}$ & $798,87 \mathbf{i}$ & Irrigada \\
\hline & & & $2,20 \mathrm{e}$ & $1,40 \mathbf{j}$ & $807,13 \mathbf{i}$ & Não irrigada \\
\hline & & \multirow{2}{*}{$3 \times 2$} & $2,95 \mathbf{d}$ & $3,67 \mathbf{j}$ & $1174,95 \mathbf{i}$ & Irrigada \\
\hline & & & $1,67 \mathrm{e}$ & $2,54 \mathbf{j}$ & $1222,33 \mathbf{i}$ & Não irrigada \\
\hline & & \multirow{2}{*}{$3 \times 1$} & $2,33 \mathbf{e}$ & $6,29 \mathbf{i}$ & $2068,23 \mathbf{h}$ & Irrigada \\
\hline & & & $2,55 \mathrm{e}$ & $4,96 \mathbf{j}$ & $2130,26 \mathbf{h}$ & Não irrigada \\
\hline & & \multirow{2}{*}{$1,5 \times 2$} & $1,61 \mathbf{e}$ & $3,96 \mathbf{j}$ & $2291,78 \mathbf{h}$ & Irrigada \\
\hline & & & $1,89 \mathrm{e}$ & $6,05 \mathbf{i}$ & $2319,04 \mathbf{h}$ & Não irrigada \\
\hline & & \multirow{2}{*}{$3 \times 0,5$} & $1,94 \mathbf{e}$ & $8,35 \mathbf{h}$ & $4356,93 \mathbf{g}$ & Irrigada \\
\hline & & & $2,58 \mathrm{e}$ & $7,09 \mathbf{i}$ & $4485,76 \mathbf{g}$ & Não irrigada \\
\hline & \multirow{10}{*}{12} & \multirow{2}{*}{$3 \times 3$} & $6,76 \mathrm{c}$ & $16,88 \mathbf{g}$ & $5368,67 \mathbf{f}$ & Irrigada \\
\hline & & & $4,83 \mathrm{c}$ & $7,97 \mathbf{h}$ & $2516,12 \mathbf{h}$ & Não irrigada \\
\hline & & \multirow{2}{*}{$3 \times 2$} & $6,37 \mathbf{a}$ & $23,02 \mathbf{f}$ & $7400,96 \mathbf{e}$ & Irrigada \\
\hline & & & $5,03 \mathrm{c}$ & $15,40 \mathbf{h}$ & $4789,64 \mathbf{f}$ & Não irrigada \\
\hline & & \multirow{2}{*}{$3 \times 1$} & $5,26 \mathbf{b}$ & $32,23 \mathrm{e}$ & $10220,21 \mathbf{d}$ & Irrigada \\
\hline & & & $4,73 \mathrm{c}$ & $24,48 \mathbf{f}$ & $7415,34 \mathbf{e}$ & Não irrigada \\
\hline & & \multirow{2}{*}{$1,5 \times 2$} & $4,59 \mathrm{c}$ & $25,25 \mathbf{f}$ & $7251,27 \mathbf{e}$ & Irrigada \\
\hline & & & $5,14 \mathrm{c}$ & $29,43 \mathrm{e}$ & $9315,08 \mathbf{d}$ & Não irrigada \\
\hline & & \multirow{2}{*}{$3 \times 0,5$} & $3,32 \mathbf{d}$ & $23,48 \mathbf{f}$ & $6794,29 \mathrm{e}$ & Irrigada \\
\hline & & & $3,50 \mathrm{~d}$ & $24,99 \mathbf{f}$ & $7052,28 \mathbf{e}$ & Não irrigada \\
\hline \multirow{20}{*}{ B } & \multirow{10}{*}{6} & \multirow{2}{*}{$3 \times 3$} & $2,97 \mathbf{d}$ & $3,17 \mathbf{j}$ & $641,43 \mathbf{i}$ & Irrigada \\
\hline & & & $3,29 \mathbf{d}$ & $2,14 \mathbf{j}$ & $608,59 \mathbf{i}$ & Não irrigada \\
\hline & & \multirow{2}{*}{$3 \times 2$} & $2,66 \mathrm{e}$ & $3,67 \mathbf{j}$ & $931,88 \mathbf{i}$ & Irrigada \\
\hline & & & $2,43 \mathrm{e}$ & $3,63 \mathbf{j}$ & $910,15 \mathbf{i}$ & Não irrigada \\
\hline & & \multirow{2}{*}{$3 \times 1$} & $2,50 \mathbf{e}$ & $5,01 \mathbf{j}$ & $2126,86 \mathbf{h}$ & Irrigada \\
\hline & & & $2,25 \mathrm{e}$ & $6,52 \mathbf{i}$ & $2103,78 \mathbf{h}$ & Não irrigada \\
\hline & & \multirow{2}{*}{$1,5 \times 2$} & $2,96 \mathbf{d}$ & $8,90 \mathbf{h}$ & $1885,03 \mathbf{h}$ & Irrigada \\
\hline & & & $2,31 \mathbf{e}$ & $8,17 \mathbf{h}$ & $1873,47 \mathbf{h}$ & Não irrigada \\
\hline & & \multirow{2}{*}{$3 \times 0,5$} & $2,34 \mathbf{e}$ & $9,87 \mathbf{h}$ & $3804,76 \mathbf{g}$ & Irrigada \\
\hline & & & $2,53 \mathrm{e}$ & $10,06 \mathbf{h}$ & $3766,51 \mathbf{g}$ & Não irrigada \\
\hline & \multirow{10}{*}{12} & \multirow{2}{*}{$3 \times 3$} & $8,13 \mathbf{a}$ & $29,68 \mathrm{e}$ & 9586,45 d & Irrigada \\
\hline & & & $6,43 \mathbf{b}$ & $17,52 \mathbf{g}$ & 5329,87 f & Não irrigada \\
\hline & & \multirow{2}{*}{$3 \times 2$} & $7,29 \mathbf{a}$ & $35,64 \mathbf{d}$ & $11505,20 \mathrm{c}$ & Irrigada \\
\hline & & & $6,25 \mathbf{b}$ & $24,43 \mathbf{f}$ & 7037,80 e & Não irrigada \\
\hline & & \multirow{2}{*}{$3 \times 1$} & $4,87 \mathrm{c}$ & $30,43 \mathbf{e}$ & $8772,74 \mathbf{d}$ & Irrigada \\
\hline & & & $5,37 \mathrm{c}$ & $37,28 \mathbf{d}$ & $10873,85 \mathrm{c}$ & Não irrigada \\
\hline & & \multirow{2}{*}{$1,5 \times 2$} & $6,16 \mathbf{b}$ & 55,02 a & $16639,35 \mathbf{a}$ & Irrigada \\
\hline & & & $3,99 \mathbf{d}$ & $49,91 \mathbf{b}$ & $15315,65 \mathbf{b}$ & Não irrigada \\
\hline & & $2 \times 0=$ & $4,01 \mathbf{d}$ & $41,32 \mathrm{c}$ & $11640,61 \mathrm{c}$ & Irrigada \\
\hline & & $3 \times 0,5$ & $5,92 \mathbf{b}$ & $40,10 \mathrm{c}$ & $11276,77 \mathrm{c}$ & Não irrigada \\
\hline
\end{tabular}

As médias seguidas pela mesma letra pertencem a um mesmo grupo pelo teste de Skott-knott em nível de 5\% de probabilidade. DAP: diâmetro da árvore a 1,30 m do solo.

( $3 \times 3 \mathrm{~m})$, em que o volume do clone $\mathrm{A}$ aos 6 e 12 meses na região irrigada foi superior 50,4 e 52,7\%, respectivamente, à região não irrigada. Comportamento semelhante foi observado para o clone $\mathrm{B}$, sendo que o volume foi $32,2 \%$ e $40,9 \%$ superior na região irrigada para as duas idades respectivamente. Observa-se que os valores 


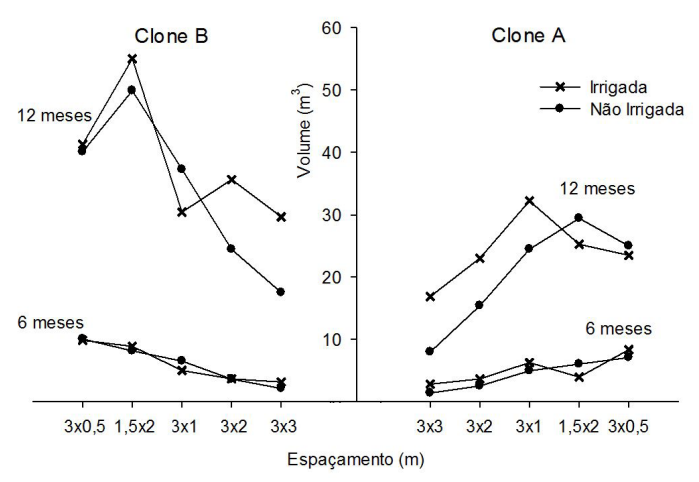

Figura 1. Valores médios de volume de madeira do fuste por hectare $\left(\mathrm{m}^{3} \cdot \mathrm{ha}^{-1}\right)$ em dois clones do híbrido E. grandis $\times E$. urophylla para diferentes, idades, espaçamentos, idades e regiões.

Figure 1. Average values of the stem wood volume per hectare $\left(\mathrm{m}^{3} \cdot \mathrm{ha}^{-1}\right)$ in two clones of the hybrid E. grandis $\times$ E. urophylla for different ages, spacings, ages and regions.

médios de massa seca por hectare seguiram a mesma tendência do volume por hectare.

O comportamento do incremento semestral $\left(\mathrm{m}^{3}\right.$.semestre $\left.{ }^{-1}\right)$ em relação aos diferentes espaçamentos e regiões pode ser visualizado na Figura 2. O incremento, assim como o volume, nos diferentes espaçamentos dos dois clones nas regiões irrigadas, foram superiores aos da região não irrigada, com exceção nos espaçamentos $3 \times 0,5$ e $1,5 \times 2 \mathrm{~m}$ do clone $\mathrm{A}$ e no espaçamento $3 \times 1 \mathrm{~m}$ do clone $\mathrm{B}$, em que o incremento foi maior na região não irrigada.

Com o aumento do espaçamento de plantio, os valores de incremento médio semestral de volume por hectare nas duas regiões decresceram. Esta mesma tendência foi observada por Oliveira et al. (2009), que avaliaram o clone do híbrido E.camadulensis $\times$ E. urophylla com idades de 18, 27, 38 e 51 meses, nos espaçamentos $3,33 \times 2 ; 3,33 \times 3$ e $5 \times 2$ m. Quenó (2009) também verificou essa tendência para o clone de um híbrido E. grandis $\times$ E. camadulensis com 2 anos nos espaçamentos $3 \times 0,5 ; 3 \times 0,75 ; 3 \times 1 ; 3 \times 1,5 ; 3 \times 2 ; 3 \times 2,5$ e $3 \times 3 \mathrm{~m}$.

\subsection{Densidade básica da madeira}

Na Tabela 2 estão os valores médios de densidade básica média ponderada dos clones nas diferentes idades, espaçamentos e regiões. Os valores médios de densidade variaram estatisticamente $(\mathrm{P}<0,05)$.

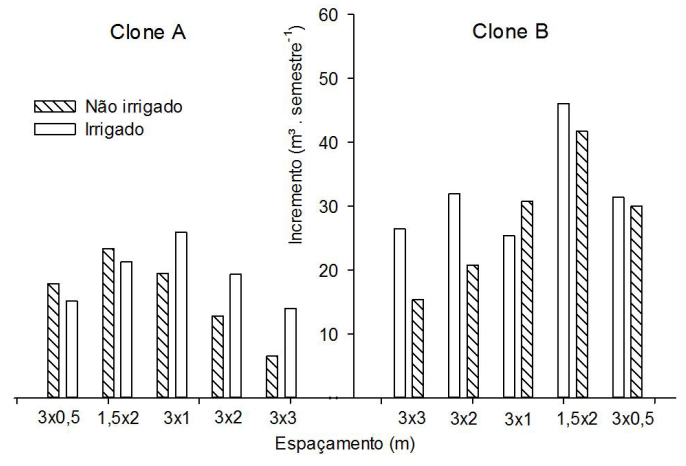

Figura 2. Incremento semestral $\left(\mathrm{m}^{3}\right.$. semestre $\left.^{-1}\right)$ em relação aos diferentes clones do híbrido E. grandis $\times$ E. urophylla, espaçamentos e regiões.

Figure 2. Half year increment $\left(\mathrm{m}^{3}\right.$. semester $\left.{ }^{-1}\right)$ in relation to different hybrid clones E. grandis $\times$ E. urophylla, spacing and regions.

Os valores médios de densidade do clone $\mathrm{A}$ foram superiores aos do clone $\mathrm{B}$, tendo os maiores valores do clone A nos espaçamentos $3 \times 3$ e $3 \times 2 \mathrm{~m}$, independente da idade e irrigação.

Conforme Benson (1963), a alta competição por água, luz e nutrientes entre as árvores de folhosas pode resultar na baixa densidade da madeira, corroborando com os resultados do presente trabalho, em que os maiores valores de densidade foram provenientes dos espaçamentos mais amplos. Ferreira et al. (1997) estudaram E. dunni com 72 meses, e Rocha (2011) o E. grandis $\times$ E. camadulensis com 85 meses; também encontraram maiores valores de densidade da madeira nos espaçamentos mais amplos. Migliorini et al. (1980) analisaram o E. urophylla com 72 meses e obtiveram resultados distintos, em que a densidade diminuiu nos maiores espaçamentos. Já Sereghetti (2012) não verificou influência do espaçamento na densidade da madeira do híbrido E. urophylla $\times$ E. grandis com 12 meses de idade.

A densidade da madeira nas duas idades para o clone A em cada região e espaçamento são ilustrados na Figura 3. O maior valor de densidade na região não irrigada foi proveniente da madeira com 6 meses, na região irrigada a densidade foi superior na idade de 12 meses, com exceção nos espaçamentos de plantio $1,5 \times 2$ e $3 \times 0,5 \mathrm{~m}$. No entanto, ao comparar os valores médios de densidade para o clone $\mathrm{A}$ entre região irrigada e não irrigada, os resultados foram iguais na grande maioria dos espaçamentos estudados. 
Tabela 2. Valores médios da densidade básica da madeira dos clones do híbrido E. grandis $\times$ E. urophylla com diferentes idades, espaçamentos e regiões.

Table 2. Mean values of wood density of hybrid clones E. grandis $\times$ E. urophylla with different ages, spacing and regions.

\begin{tabular}{|c|c|c|c|c|}
\hline Clone & Idade (meses) & Espaçamento (m) & Densidade $\left(\mathrm{g} \cdot \mathrm{cm}^{-3}\right)$ & Região \\
\hline \multirow{20}{*}{ A } & \multirow{10}{*}{6} & \multirow{2}{*}{$3 \times 3$} & $0,40 \mathbf{a}$ & Irrigada \\
\hline & & & $0,40 \mathbf{a}$ & Não irrigada \\
\hline & & \multirow{2}{*}{$3 \times 2$} & $0,39 \mathbf{a}$ & Irrigada \\
\hline & & & $0,41 \mathbf{a}$ & Não irrigada \\
\hline & & \multirow{2}{*}{$3 \times 1$} & $0,34 \mathbf{b}$ & Irrigada \\
\hline & & & $0,39 \mathbf{a}$ & Não irrigada \\
\hline & & \multirow{2}{*}{$1,5 \times 2$} & $0,38 \mathbf{a}$ & Irrigada \\
\hline & & & $0,39 \mathbf{a}$ & Não irrigada \\
\hline & & \multirow{2}{*}{$3 \times 0,5$} & $0,36 \mathbf{b}$ & Irrigada \\
\hline & & & $0,37 \mathbf{a}$ & Não irrigada \\
\hline & \multirow{10}{*}{12} & \multirow{2}{*}{$3 \times 3$} & $0,41 \mathbf{a}$ & Irrigada \\
\hline & & & $0,40 \mathbf{a}$ & Não irrigada \\
\hline & & \multirow{2}{*}{$3 \times 2$} & $0,40 \mathbf{a}$ & Irrigada \\
\hline & & & $0,38 \mathbf{a}$ & Não irrigada \\
\hline & & \multirow{2}{*}{$3 \times 1$} & $0,38 \mathbf{a}$ & Irrigada \\
\hline & & & $0,37 \mathbf{b}$ & Não irrigada \\
\hline & & \multirow{2}{*}{$1,5 \times 2$} & $0,35 \mathbf{b}$ & Irrigada \\
\hline & & & $0,39 \mathbf{a}$ & Não irrigada \\
\hline & & \multirow{2}{*}{$3 \times 0,5$} & $0,35 \mathbf{b}$ & Irrigada \\
\hline & & & $0,35 \mathbf{b}$ & Não irrigada \\
\hline \multirow{20}{*}{ B } & \multirow{10}{*}{6} & \multirow{2}{*}{$3 \times 3$} & $0,35 \mathbf{b}$ & Irrigada \\
\hline & & & $0,33 \mathbf{b}$ & Não irrigada \\
\hline & & \multirow{2}{*}{$3 \times 2$} & $0,34 \mathbf{b}$ & Irrigada \\
\hline & & & $0,33 \mathbf{b}$ & Não irrigada \\
\hline & & \multirow{2}{*}{$3 \times 1$} & $0,39 \mathbf{a}$ & Irrigada \\
\hline & & & $0,35 \mathbf{b}$ & Não irrigada \\
\hline & & \multirow{2}{*}{$1,5 \times 2$} & $0,34 \mathbf{b}$ & Irrigada \\
\hline & & & $0,34 \mathbf{b}$ & Não irrigada \\
\hline & & \multirow{2}{*}{$3 \times 0,5$} & $0,34 \mathbf{b}$ & Irrigada \\
\hline & & & $0,34 \mathbf{b}$ & Não irrigada \\
\hline & \multirow{10}{*}{12} & \multirow{2}{*}{$3 \times 3$} & $0,36 \mathbf{b}$ & Irrigada \\
\hline & & & $0,35 \mathbf{b}$ & Não irrigada \\
\hline & & \multirow{2}{*}{$3 \times 2$} & $0,37 \mathbf{b}$ & Irrigada \\
\hline & & & $0,34 \mathbf{b}$ & Não irrigada \\
\hline & & \multirow{2}{*}{$3 \times 1$} & $0,34 \mathbf{b}$ & Irrigada \\
\hline & & & $0,34 \mathbf{b}$ & Não irrigada \\
\hline & & \multirow{2}{*}{$1,5 \times 2$} & $0,34 \mathbf{b}$ & Irrigada \\
\hline & & & $0,34 \mathbf{b}$ & Não irrigada \\
\hline & & \multirow{2}{*}{$3 \times 0,5$} & $0,34 \mathbf{b}$ & Irrigada \\
\hline & & & $0,35 \mathbf{b}$ & Não irrigada \\
\hline
\end{tabular}

As médias seguidas pela mesma letra pertencem a um mesmo grupo pelo teste de Skott-knott em nível de 5\% de probabilidade.

$\mathrm{Na}$ Figura 4 podem ser visualizados os valores de densidade entre as idades do clone $\mathrm{B}$ em cada região e espaçamento. Ao analisar em números absolutos, aos 12 meses a densidade da madeira foi superior na região não irrigada, com exceção nos espaçamentos $1,5 \times 2$ e $3 \times 1 \mathrm{~m}$. Na região irrigada, os maiores valores foram provenientes dos espaçamentos $3 \times 3$ e $3 \times 2 \mathrm{~m}$ com 12 meses e no espaçamento $3 \times 1$ m com 6 meses; 


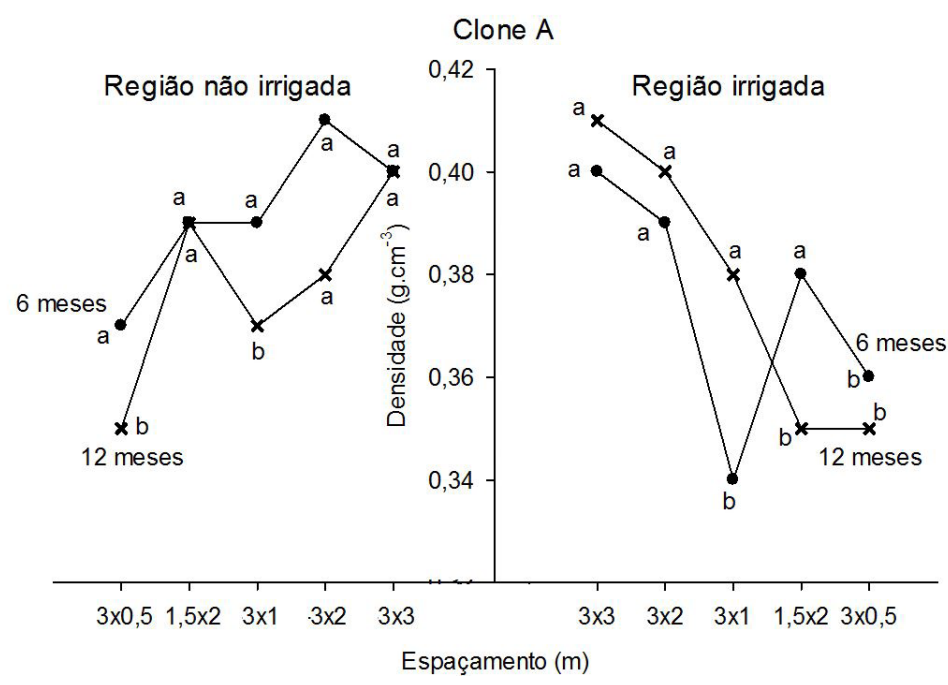

Figura 3. Valores médios de densidade da madeira do clone A do híbrido E. grandis $\times$ E. urophylla nos diferentes espaçamentos e idades, na região irrigada e não irrigada.

Figure 3. Average values of wood density of clone A hybrid E. grandis $\times$ E. urophylla in different ages and spacings in the region and without irrigation.

\section{Clone B}

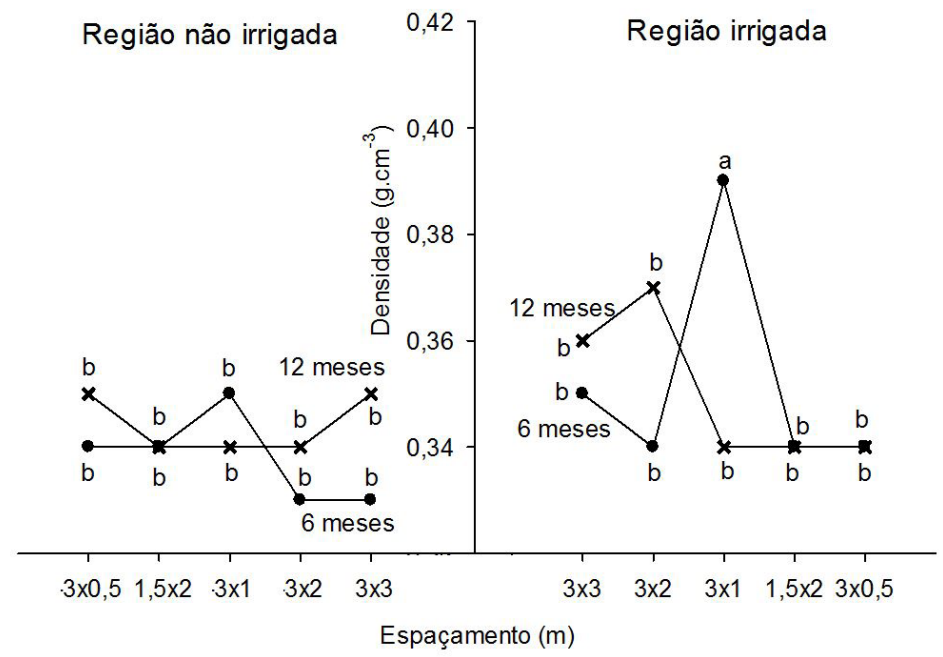

Figura 4. Valores médios de densidade da madeira do clone B do híbrido E. grandis $\times$ E. urophylla nos diferentes espaçamentos e idades, na região irrigada e não irrigada.

Figure 4. Average values of wood density of the hybrid clone B E. grandis $\times$ E. urophylla in different ages and spacings in the region and without irrigation.

no restante dos espaçamentos, as densidades foram semelhantes.

Ao verificar a densidade da madeira do clone $B$ nas duas regiões, observou-se que na região irrigada foram obtidos os maiores valores médios (Figura 4). Embora
Downes et al. (2006) tenham estudado a densidade da madeira do Eucalyptus globulus aos 8 anos e obtido resultado inverso ao do clone $\mathrm{B}$, sendo a densidade inferior na região irrigada. Já Drew et al. (2009) não observaram diferença da densidade na madeira 
avaliada mensalmente no período de 33 a 57 meses do E. globulus entre a região irrigada e não irrigada. Pereira \& Araújo (1990) encontraram resultado inferior de densidade na região irrigada para a madeira de E. globulus com 11 meses de idade, e nessa mesma espécie com idade de seis meses foi obtido resultado inverso, em que a densidade foi maior na região irrigada.

O efeito do adensamento do espaçamento de plantio no volume e densidade básica da madeira pode ser visualizado na Tabela 3. Verificou-se maior efeito do espaçamento de plantio no volume de madeira, sendo essa variação maior na região não irrigada, ou seja, a diferença do volume de madeira entre o menor $(3 \times 0,5 \mathrm{~m})$ e maior $(3 \times 3 \mathrm{~m})$ espaçamento foi superior na região não irrigada. Isso foi ocasionado pelo maior desenvolvimento das plantas nos maiores espaçamentos da região irrigada, em contrapartida esse mesmo desenvolvimento não ocorreu nos menores espaçamentos em função do menor espaço útil para o desenvolvimento da planta. A menor variação do volume de madeira ocorreu nos clones aos 12 meses de idade na região irrigada, com valor em torno de $28 \%$.

Como pode ser visualizado na Tabela 3 , a densidade da madeira diminuiu com o adensamento de plantio, e na madeira com idade de 6 meses foi possível identificar maior variação, contudo a redução ainda foi superior na região irrigada. A pequena variação da densidade no clone B resultou na igualdade estatística entre os valores do menor e maior espaçamento.

\section{CONCLUSÕES}

Com base nos resultados obtidos, observa-se que as idades, espaçamentos e regiões influenciaram no volume e densidade básica da madeira. E os maiores valores de volume de madeira por hectare foram obtidos nos espaçamentos mais densos e na região irrigada.

Tabela 3. Variação dos valores médios da densidade básica e volume da madeira dos clones do híbrido E. grandis $\times$ E. urophylla com o adensamento do espaçamento.

Table 3. Variation of the average values of the density and volume of the wood of hybrid clones E. grandis $\times$ E. urophylla with the density spacing.

\begin{tabular}{|c|c|c|c|c|c|c|c|c|c|}
\hline \multirow{2}{*}{$\begin{array}{l}\text { Clone } \\
\text { (Idade) }\end{array}$} & \multirow[b]{2}{*}{$\operatorname{Esp}(\mathbf{m})$} & \multicolumn{4}{|c|}{ Região Irrigada } & \multicolumn{4}{|c|}{ Região Não Irrigada } \\
\hline & & $\begin{array}{c}\text { Dbp } \\
\left(\mathrm{g} \mathrm{cm}^{-3}\right)\end{array}$ & $\begin{array}{l}\text { Var } \\
(\%)^{*}\end{array}$ & $\begin{array}{c}\text { Vol } \\
\left(\mathrm{m}^{3}\right)\end{array}$ & $\begin{array}{l}\text { Var } \\
(\%)\end{array}$ & $\begin{array}{c}\text { Dbp } \\
\left(\mathrm{g} \mathrm{cm}^{-3}\right)\end{array}$ & $\operatorname{Var}(\%)$ & $\begin{array}{c}\text { Vol } \\
\left(\mathrm{m}^{3}\right)\end{array}$ & $\operatorname{Var}(\%)$ \\
\hline \multirow{5}{*}{$\begin{array}{c}\text { Clone A } \\
\text { (6 meses) }\end{array}$} & $3 \times 3$ & 0,40 & \multirow{5}{*}{$\downarrow 10,00$} & 2,84 & \multirow{5}{*}{$\uparrow 66,00$} & 0,40 & \multirow{5}{*}{$\downarrow 7,50$} & 1,41 & \multirow{5}{*}{$\uparrow 80,16$} \\
\hline & $3 \times 2$ & 0,39 & & 3,67 & & 0,41 & & 2,55 & \\
\hline & $3 \times 1$ & 0,34 & & 6,30 & & 0,39 & & 4,96 & \\
\hline & $1,5 \times 2$ & 0,38 & & 3,96 & & 0,39 & & 6,06 & \\
\hline & $3 \times 0,5$ & 0,36 & & 8,35 & & 0,37 & & 7,10 & \\
\hline \multirow{5}{*}{$\begin{array}{c}\text { Clone } \\
\text { A (12 } \\
\text { meses) }\end{array}$} & $3 \times 3$ & 0,41 & \multirow{5}{*}{$\downarrow 14,63$} & 16,88 & \multirow{5}{*}{$\uparrow 28,12$} & 0,40 & \multirow{5}{*}{$\downarrow 12,50$} & 7,98 & \multirow{5}{*}{$\uparrow 68,08$} \\
\hline & $3 \times 2$ & 0,40 & & 23,02 & & 0,38 & & 15,40 & \\
\hline & $3 \times 1$ & 0,38 & & 32,24 & & 0,37 & & 24,48 & \\
\hline & $1,5 \times 2$ & 0,35 & & 25,25 & & 0,39 & & 29,44 & \\
\hline & $3 \times 0,5$ & 0,35 & & 23,49 & & 0,35 & & 24,99 & \\
\hline \multirow{5}{*}{$\begin{array}{c}\text { Clone B } \\
\text { (6 meses) }\end{array}$} & $3 \times 3$ & 0,35 & \multirow{5}{*}{$\downarrow 2,86$} & 3,17 & \multirow{5}{*}{$\uparrow 67,88$} & 0,33 & \multirow{5}{*}{$\uparrow 3,03$} & 2,14 & \multirow{5}{*}{$\uparrow 78,72$} \\
\hline & $3 \times 2$ & 0,34 & & 3,67 & & 0,33 & & 3,63 & \\
\hline & $3 \times 1$ & 0,39 & & 5,01 & & 0,35 & & 6,52 & \\
\hline & $1,5 \times 2$ & 0,34 & & 8,90 & & 0,34 & & 8,17 & \\
\hline & $3 \times 0,5$ & 0,34 & & 9,87 & & 0,34 & & 10,06 & \\
\hline \multirow{5}{*}{$\begin{array}{c}\text { Clone } \\
\text { B (12 } \\
\text { meses) }\end{array}$} & $3 \times 3$ & 0,36 & \multirow{5}{*}{$\downarrow 5,56$} & 29,68 & \multirow{5}{*}{$\uparrow 28,17$} & 0,35 & \multirow{5}{*}{$\downarrow 2,86$} & 17,52 & \multirow{5}{*}{$\uparrow 56,30$} \\
\hline & $3 \times 2$ & 0,37 & & 35,64 & & 0,34 & & 24,43 & \\
\hline & $3 \times 1$ & 0,34 & & 30,43 & & 0,34 & & 37,28 & \\
\hline & $1,5 \times 2$ & 0,34 & & 55,02 & & 0,35 & & 49,91 & \\
\hline & $3 \times 0,5$ & 0,34 & & 41,32 & & 0,34 & & 40,10 & \\
\hline
\end{tabular}

Esp: espaçamento; Dbp: densidade básica ponderada; Vol: volume; Var: Variação; ${ }^{*}$ Variação analisada do maior para o menor espaçamento de plantio. 
Os resultados de densidade básica para os dois clones nas duas idades foram iguais entre região irrigada e não irrigada na grande maioria dos espaçamentos estudados.

A influência do espaçamento na densidade básica da madeira foi verificada para o clone $A$, uma vez que nos espaçamentos mais amplos houve maiores valores. No entanto, no clone B não foi observada influência significativa do espaçamento na densidade básica.

O volume de madeira sofreu maior variação com o adensamento do espaçamento, em que essa variação foi ainda mais significativa na região não irrigada. A variação da densidade foi baixa, principalmente no clone B.

\section{STATUS DA SUBMISSÃO}

Recebido: 14 jan., 2014

Aceito: 26 jun., 2016

\section{AUTOR(ES) PARA CORRESPONDÊNCIA}

\section{Jordão Cabral Moulin}

Departamento de Ciências Florestais e da Madeira - DCFM, Universidade Federal do Espírito Santo - UFES, Av. Gov. Lindemberg, 316, Centro, CEP 29500-000, Jeronimo Monteiro, ES, Brasil

e-mail: jordao_cm@hotmail.com

\section{REFERENNCIAS}

Associação Brasileira de Normas Técnicas - ABNT. NBR 11941: madeira: determinação da densidade básica. Rio de Janeiro: ABNT; 2003. 6 p.

Balloni EA, Simões JW. O espaçamento de plantio e suas implicações silviculturais. Instituto de Pesquisas e Estudos Florestais 1980; 1(3): 1-16.

Benson HP. The application of silviculture in controlling the specific gravity of wood. Estados Unidos: Forest Service; 1963. Technical Bulletin of United States Department of Agriculture n. 1288.

Berger R, Schneider PR, Finger CAG, Haselein CR. Efeito do espaçamento e da adubação no crescimento de um clone de Eucalyptus saligna Smith. Ciência Florestal 2002; 12(2): 75-87. http://dx.doi.org/10.5902/198050981682.

Brasil MAM, Ferreira M. Variação da densidade básica da madeira de Eucalyptus alba Reinw, E. saligna Smith e E. grandis Hill Ex-maiden aos 5 anos de idade, em função do local e do espaçamento. Instituto de Pesquisas e Estudos Florestais 1971; (2-3): 129-149.

Cardoso A Jr. Bracatinga. Brasil Madeira 1989; 3(33): 1-10. Coelho ASR, Mello HÁ, Simões JW. Comportamento de espécies de eucaliptos face ao espaçamento. Instituto de Pesquisas e Estudos Florestais 1970; 2-3(50): 129-149.

Downes G, Worledge D, Schimleck L, Harwood C, French J, Beadle C. The effect of growth rate and irrigation on the basic density and kraft pulp yield of Eucalyptus globulus and E. nitens. New Zealand Journal of Forestry 2006; 51(3): 13-22.

Drew DM, Downes GM, O'grady AP, Read J, Worledge D. High resolution temporal variation in wood properties in irrigated and non-irrigated Eucalyptus globules. Annals of Forest Science 2009; 406(66): 1-10.

Ferreira GW, Gonzaga JV, Foelkel CEB, Assis TF, Ratnieks E, Silva MCM. Qualidade da celulose kraft-antraquinona de Eucalyptus dunnii plantado em cinco espaçamentos em relação ao Eucalyptus grandis e Eucalyptus saligna. Ciência Florestal 1997; 7(1): 41-63. http://dx.doi. org/10.5902/19805098338.

FIBRIA. Produção de biomassa energética em diferentes espaçamentos, manejos e culturas: eucalipto - dados de análise internos da Empresa Fibria [online]. mensagem para: Jordão Cabral Moulin. 2013 Set 20 [citado em 2014 Jan 13].

Haygreen JG, Bowyer JL. Forest products and wood science. 2rd ed. Ames: Iowa State University; 1989.

Mello HA, Simões JW, Ferreira CA, Brasil UM. Influência do espaçamento e da idade de corte na produção de madeira de eucalipto em solo de cerrado. Instituto de Pesquisa e Estudos Florestais 1976; 13(1): 143-162.

Migliorini AJ, Brito JO, Barrichelo LEG. Influência das práticas silviculturais na produção de carvão vegetal. Piracicaba: Instituto de Pesquisas e Estudos Florestais; 1980. Circular Técnica n. 104.

Morais VM. Dinâmica de crescimento de eucalipto clonal sob diferentes espaçamentos, na região noroeste do Estado de Minas Gerais [dissertação]. Lavras: Universidade Federal de Lavras; 2006.

Müller MD, Couto L. Avaliação de densidades de plantio e rotação de plantações de rápido crescimento para produção de biomassa. Viçosa: Renabio; 2006. 65 p. Documento técnico n. 2.

Oliveira TK, Macedo RLG, Venturin N, Higashikawa EM. Desempenho silvicultural e produtivo de eucalipto sob diferentes arranjos espaciais em sistema agrossilvipastoril. Pesquisa Florestal Brasileira 2009; (60): 1-10. http://dx.doi.org/10.4336/2009.pfb.60.01.

Pereira $\mathrm{H}$, Araújo C. Raw-material quality of fast grown Eucalyptus globules during the first year. IAWA Bulletin 1990; 11(4): 421-427. http://dx.doi.org/10.1163/2294193290000532 . 
Quenó LRM. Viabilidade econômica da produção de biomassa de eucalipto e de capim elefante para energia [dissertação]. Brasília: Universidade de Brasília; 2009.

Rocha MFV. Influência do espaçamento e da idade na produtividade e propriedades da madeira de Eucalyptus grandis $\times$ Eucalyptus camaldulensis para energia [dissertação]. Viçosa: Universidade Federal de Viçosa; 2011.

Schonau APG, Coetzee J. Initial spacing, stand density and thinning in eucalypt plantations. Forest Ecology and Management 1989; 29: 245-266.
Sereghetti GC. Biomassa inicial do híbrido Eucalyptus urophylla vs. Eucalyptus grandis em diferentes espaçamentos [dissertação]. Botucatu: Universidade Estadual de São Paulo; 2012.

Tomazello M Fo. Efeito da irrigação e fertilização nas propriedades do lenho de árvores de Eucalyptus grandis $\times$ urophylla [tese]. Piracicaba: Escola Superior de Agricultura Luiz de Queiroz; 2006.

Wimmer R, Evans R, Downes GM, Rasmussen G, French J. Direct effects of wood characteristics on pulp and handsheet properties of Eucalyptus globulus. Holzforschung 2002; 2002(56): 244-252. 